\title{
Measuring interface electrostatic potential and surface charge in a scanning electron microscope
}

\author{
I. Sychugov, ${ }^{\text {a) }}$ Y. Nakayama, and K. Mitsuishi \\ Quantum Dot Research Center, National Institute for Materials Science, Sakura 3-13, Tsukuba, \\ Ibaraki 305-0003, Japan
}

(Received 11 June 2009; accepted 28 September 2009; published 30 October 2009)

\begin{abstract}
A novel method for electrostatic potential measurements at the interface is described. It involves placing a two-dimensional grid below the sample and observing it in a scanning electron microscope. Primary electron beam displacement, caused by surface charges, can be then measured for every grid knot. Using geometric parameters of the setup, a quantitative mapping of the potential can be extracted. It is shown that this method can achieve a tens of millivolt sensitivity and a submicron spatial resolution in electrostatic potential measurements. () 2009 American Vacuum Society. [DOI: 10.1116/1.3253475]
\end{abstract}

\section{INTRODUCTION}

A scanning electron microscope (SEM) is a versatile tool widely used for material observation mainly through the secondary electron emission process. It was shown that the emission sensitivity to the material work function can be used to obtain electrostatic potential distribution inside doped semiconductor devices. ${ }^{1}$ A higher spatial resolution, though, can be achieved in a transmission electron microscope (TEM) using off-axis electron holography. ${ }^{2}$ With this method, a two-dimensional potential distribution inside a $0.18 \mu \mathrm{m}$ transistor was shown. ${ }^{3}$ However, whether it is a secondary emission process or a phase shift in the transmitted beam, these methods require a substance for the signal generation. On the other hand, electric field strength in the vicinity of a material was obtained for a tungsten emitter inside a TEM. ${ }^{4}$ It was recently shown that this method is also feasible for a SEM, limited, however, to a high extraction bias of more than $200 \mathrm{~V}^{5}$

In this article, a method for the two-dimensional electrostatic potential mapping at the interface with a sensitivity down to tens of millivolts and a resolution in the submicron range is described. Sample surface was charged with a primary electron beam and the electric potential distribution of this charge was measured in a SEM. Quantitative analyses of the technique as well as its limitations are discussed. Possible applications of this method include the investigation of surface structure electric properties and the direct visualization of the potential distribution inside gaps. In addition, charges on the surface can also be detected and quantitatively evaluated by this technique.

\section{EXPERIMENTAL DETAILS AND RESULTS}

We used a scanning electron microscope JSM 7000F with a thermal field emission gun (JEOL). A high beam current generated by this machine was found advantageous for the charging used in the experiment. A copper mesh with a $12.5 \mu \mathrm{m}$ pitch and a square grating replica with 2000

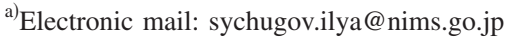

lines/mm (Oken Shoji) were employed as a reference. Samples were prepared from a silicon-on-insulator (SOI) wafer with a $1-\mu \mathrm{m}$-thick dioxide layer and a $6-\mu \mathrm{m}$-thick top silicon layer (E\&M Corp.). The thickness of the latter was tailored by the reactive ion etching process using $\mathrm{SF}_{6}$ gas (ULVAC CE-3001). Prior to the observation chip edges were polished by a set of diamond lapping films with the finest film of $\sim 0.3 \mu \mathrm{m}$ grade. The sample was usually inclined several degrees to make the grid visible for the deflected primary beam (cf. Fig. 1).

Results are shown in Figs. 2(a) and 3(a). A sample edge and a grid are visible on each figure. First, a rough grid with a $12.5 \mu \mathrm{m}$ pitch was used and the SOI chip had no Si top layer, leaving $\sim 1-\mu \mathrm{m}$-wide $\mathrm{SiO}_{2}$ layer exposed [Fig. 2(a)]. An area in the middle of the figure had been observed for some time before this demagnified image was recorded. The grid image looks distorted due to the induced charge at the sample edge, as described below. White vectors show beam displacement $D$ from the recorded knot position to the real position of the each grid knot (shown by black crosses). The real positions are obtained by the extrapolation of the grid pattern from the area unaffected by the charge. Recalculated values of the potential from the measured beam displacement are shown in Fig. 2(b). However, as seen in Fig. 2(a) for this grid, a $5 \mu \mathrm{m}$ rib width and a strong beam displacement make the determination of the recorded knot positions somewhat ambiguous. To circumvent this problem, a finer mesh with a $0.5 \mu \mathrm{m}$ pitch and very narrow ribs was used, as shown in Fig. 3(a). In this experiment, a SOI chip with a $\sim 120 \mathrm{~nm}$ Si top layer was observed. Reconstructed potential distribution is shown in Fig. 3(b), where a spline smoothing was applied to the extracted 119 knot values of the electrostatic potential.

For a low energy primary beam (here $\varepsilon=1000 \mathrm{~V}$ ), the secondary electron yield in $\mathrm{SiO}_{2}$ is relatively high: $\delta \approx 3 .{ }^{6}$ The local charge balance in the sample consists of the incoming electrons on the one hand and the backscattered, secondary, and leaked to the ground electrons on the other. When the latter prevails the net charge $Q$ becomes positive, 


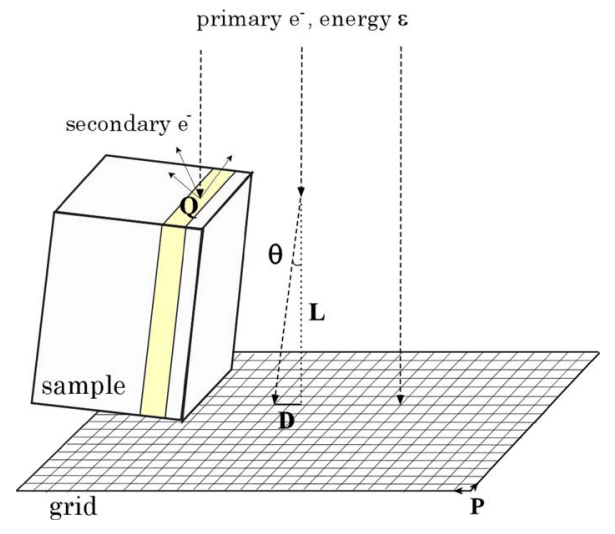

FIG. 1. (Color online) Schematic representation of the experiment configuration. A silicon-on-insulator chip with a polished top edge is mounted on top of a grid with a pitch $P$. When the primary electron beam with energy $\varepsilon$ scans across the sample, it charges the insulating layer positively via secondary electron emission with a charge $Q$. This charge deflects trajectories of the primary electrons traveling close to the sample edge, shifting them from the initial path by the value of displacement $D$, measured at the grid plane. The interaction of the primary beam with the charge $Q$ can be considered as a single scattering event at the sample-grid separation distance $L$, where $L \gg D$.
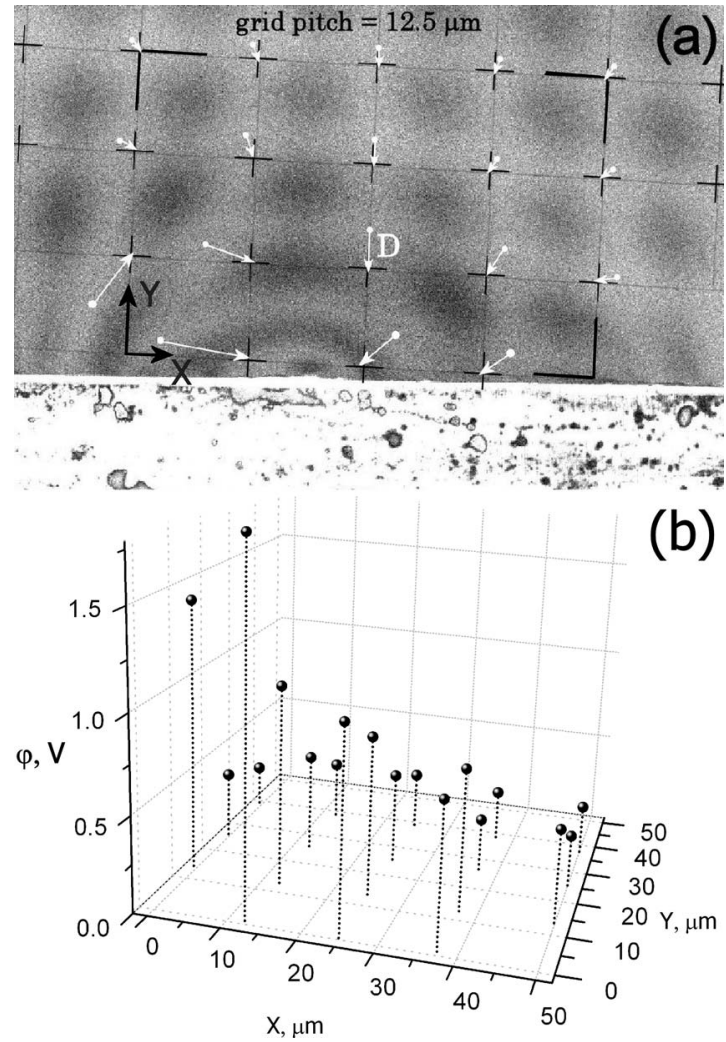

FIG. 2. (a) SEM image of a rough grid with a $12.5 \mu \mathrm{m}$ pitch. The sample is a SOI chip with completely etched top $\mathrm{Si}$ layer, exposing a $1 \mu \mathrm{m} \mathrm{SiO}$ layer, seen as a bright stripe. A charged area in the middle, affecting beam trajectories at the interface, is clearly visible. (b) Electric potential values recalculated from the measured knot displacement using Rutherford scattering approximation.
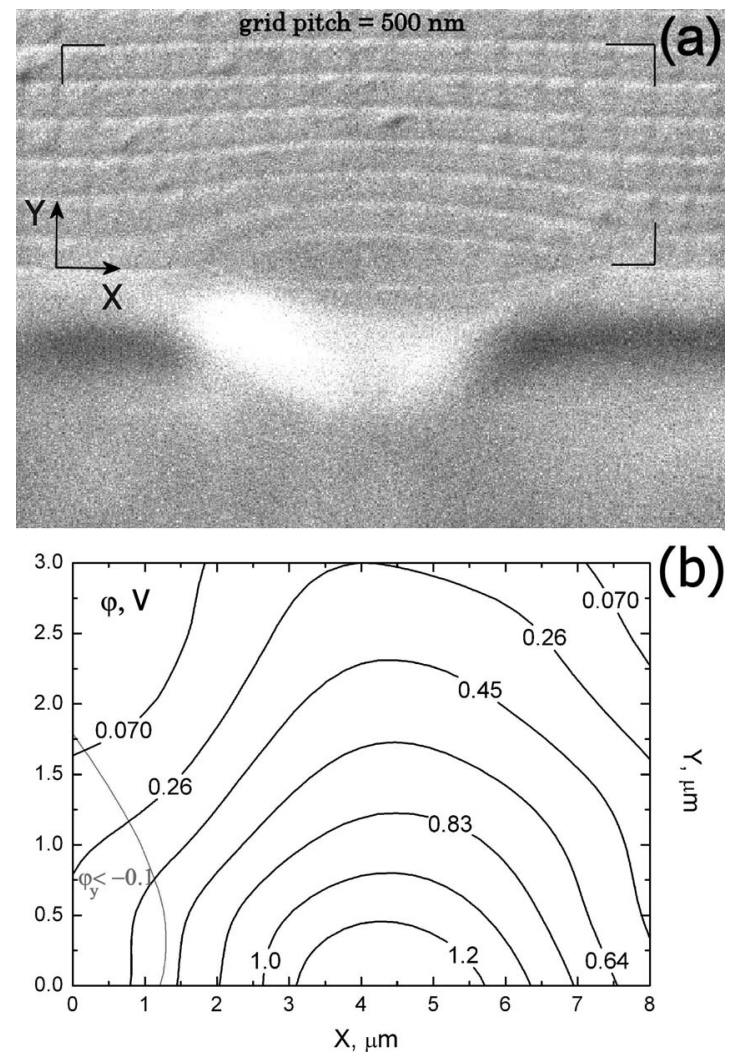

FIG. 3. (a) SEM image of a fine grid with a $0.5 \mu \mathrm{m}$ pitch. The sample is a SOI chip with a $120 \mathrm{~nm}$ top $\mathrm{Si}$ layer and a $1 \mu \mathrm{m} \mathrm{SiO}$ layer, seen as a dark stripe. A $17 \times 7$ array of the displacement values can be measured within the area designated by dark arrows. (b) A potential map of the $8 \times 3 \mu \mathrm{m}^{2}$ area obtained using a spline smoothing function. A negative $y$-component potential region at the left bottom of the plot is shown.

$$
I_{\text {beam }}<(\eta+\delta) \cdot I_{\text {beam }}+I_{\text {drain }},
$$

where $\eta$ is a backscattered electrons coefficient, $I_{\text {beam }}$ is a primary beam current, and $I_{\text {drain }}$ is a current to the ground. Thus high values of the secondary electron yield of $\mathrm{SiO}_{2}$ can be used for controllable local charging. For that, first, an area at the sample edge was zoomed in and the beam was left scanning for some time. Then the beam was scanned over a larger area to record beam displacement $D$ due to the induced charge $Q$ (Fig. 1). Such a process of local charging with subsequent larger scale observation was used, for example, in dielectric flashover studies ${ }^{7}$ and sometimes referred to as an electrostatic mirror. ${ }^{8}$

\section{DISCUSSION}

We consider interaction of the primary beam with the induced charge $Q$ as a single scattering event similar to that of Rutherford scattering. An impinging electron with impact parameter $b$ and energy $\varepsilon$ has its trajectory deflected by the angle $\theta$, such that

$$
b=\frac{k Q e}{2 \varepsilon} \cot \left(\frac{\theta}{2}\right),
$$

where $k$ is Coulomb constant. Using trigonometric formulas and the fact that the sample-grid distance much larger than 
the beam displacement $L \gg D$ (cf. Fig. 1) one can deduce the charge $Q$ as

$$
Q=\frac{\varepsilon b D}{k e L} \text {. }
$$

Then the electric potential $\varphi$ can be expressed as

$$
\varphi=k \frac{Q}{b} \equiv \frac{D}{L} \varepsilon .
$$

Thus, knowing the primary beam energy $\varepsilon$ and the samplegrid distance $L$, it is possible to obtain electric potential values $\varphi$ by simply measuring the beam displacement $D$ [calculation results are shown in Figs. 2(b) and 3(b)]. The smallest extracted value of the potential from Fig. 3(a) is $\sim 20 \mathrm{mV}$, corresponding to the minimal measured beam displacement of $\sim 60 \mathrm{~nm}$, roughly a one-tenth of the pitch size.

To measure the beam displacement clearly the grid and the sample need to be in focus simultaneously. In other words, two nearest grid knots need to be resolved while the sample is not completely out of focus yet. From the SEM geometry one can express the depth of focus DF through the microscope objective lens aperture diameter $A$ and the working distance WD as a function of the desired pixel size, which is here the grid pitch size $P$

$$
\mathrm{DF}=\frac{2 \mathrm{WD}}{A} P \text {. }
$$

And the sample-grid distance $L$ should be within this range: $L<\mathrm{DF}$.

This condition puts a practical limit on the highest spatial resolution achievable with this method. While a finer grid with a smaller $P$ is readily available, it inevitably restricts usable sample height. Here, for example, in order to have the grid with a $0.5 \mu \mathrm{m}$ pitch in focus the sample had to be polished down to $300 \mu \mathrm{m}$ [cf. Fig. 3(a)]. At the same time for the grid with a $12.5 \mu \mathrm{m}$ pitch, a $5 \mathrm{~mm}$ sample-grid separation still allows simultaneous observation [Fig. 2(a)].

In Fig. 3(b), at the left bottom part, the region of negative values for the potential $y$-component $\varphi_{y}$ is shown. These values were obtained from the displacement $y$-component $D_{y}$ in the same way as potential absolute values $\varphi$ from the measured displacement $D$. A change in the sign of $\varphi_{y}$ implies a transition from the positive to the negative sign of the charge $Q$ on the sample. This effect can also be noticed in Fig. 2(a) for the lowest left grid knot. We suggest that the area close to the intentionally charged region can accumulate electric image charge, sufficient for the measurable primary beam displacement.

In Fig. 4 we show results of the numerical simulation of the potential distribution [Fig. 4(a)] for a given surface charge profile [Fig. 4(b)]. The results were obtained by solving a two-dimensional (2D) Poisson equation using a finite element method. Obtained charged distribution represents the equivalent linear charge at the interface. Although in the present experiment the charged area had some depth and this representation is only an approximation, for the charges distributed on the sample surface the result would be more
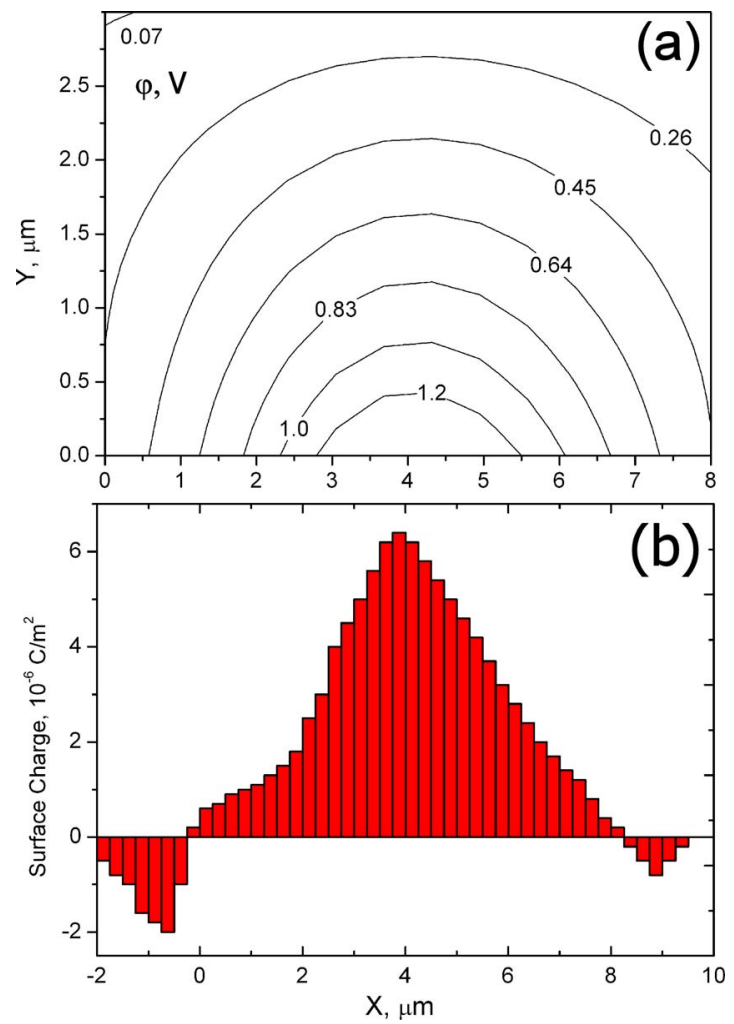

FIG. 4. (Color online) (a) Solution of the 2D Poisson equation for the surface charge distribution shown in (b). The charge profile yielding potential distribution close to the experimentally measured (Fig. 3) is shown. Note regions of the negative charge around the main area.

straightforward. Indeed, it is seen that the negative charge around the main area is necessary to obtain potential distribution similar to the experimentally measured (Fig. 3). A mismatch in the isopotential curves for smaller potentials can be attributed to the uncertainty of the small displacement measurement.

The electron beam charging as a method for the electrostatic potential excitation was chosen here merely for convenience. In general, a separate electric excitation can be used just as well, not limiting measurable systems to the ones with insulating parts. On the other hand, with this particular method a surface structure response to the electric field can be directly visualized in a SEM.

\section{CONCLUSION}

We have introduced a method for the mapping of the electrostatic potential at the interface in a scanning electron microscope. A SOI chip edge was charged by the beam and changes in the adjacent beam trajectories hitting a grid target were recorded. Experimentally measured beam displacement values were recalculated to the electrostatic potential using a Rutherford scattering model. It is shown that a mapping with a sensitivity of tens of millivolts and a submicron spatial resolution can be achieved by this method. 


\section{ACKNOWLEDGEMENTS}

This research was partially supported by Ministry of Education, Science, Sports and Culture (MEXT) through a Grant-in-Aid for Scientific Research (C) (Grant No. 21560036), and also partially supported by the "Nuclear Research Project" and "Nanotechnology Network Project" of MEXT.

${ }^{1}$ B. Kaestner, C. Schonjahn, and C. J. Humphreys, Appl. Phys. Lett. 84, 2109 (2004).

${ }^{2}$ M. R. McCartney, D. J. Smith, R. Hull, J. C. Bean, E. Voelkl, and B.
Frost, Appl. Phys. Lett. 65, 2603 (1994).

${ }^{3}$ W. D. Rau, P. Schwander, F. H. Baumann, W. Hoppner, and A. Ourmazd, Phys. Rev. Lett. 82, 2614 (1999).

${ }^{4}$ J.-I. Fujita, Y. Ikeda, S. Okada, K. Higashi, S. Nakasawa, M. Ishida, and S. Matsui, J. Vac. Sci. Technol. B 25, 2624 (2007).

${ }^{5}$ J.-I. Fujita, Y. Ikeda, and I. Suzuki, J. Vac. Sci. Technol. B 26, 2069 (2008).

${ }^{6}$ Y. C. Yong, J. T. L. Thong, and J. C. H. Phang, J. Appl. Phys. 84, 4543 (1998).

${ }^{7}$ C. Le Gressus, F. Valin, M. Henriot, M. Gautier, J. P. Duraud, T. S. Sudarshan, R. G. Bommakanti, and G. Blaise, J. Appl. Phys. 69, 6325 (1991).

${ }^{8}$ J. Liebault, K. Zarbout, G. Moya, and A. Kallel, J. Non-Cryst. Solids 322, 213 (2003). 